\title{
Smart Grids: A New Framework for Efficient Power Management in Datacenter Networks
}

\author{
Okafor Kennedy .C, Udeze Chidiebele. C \\ ${ }^{2,3}$ R \& D Department, Electronics Development Institute \\ (FMST-NASENI), Awka, Nigeria. \\ ${ }^{1}$ Electrical Electronics Engineering, Federal University of \\ Technology Owerri,, Nigeria.
}

\author{
E. C. N. Okafor, C. C. Okezie, \\ ${ }^{4}$ Electronics and Computer Engineering Department, Nnamdi \\ Azikiwe University, Awka, Nigeria.
}

\begin{abstract}
The energy demand in the enterprise market segment demands a supply format that accommodates all generation and storage options with active participation by end users in demand response. Basically, with today's high power computing (HPC), a highly reliable, scalable, and cost effective energy solution that will satisfy power demands and improve environmental sustainability will have a broad acceptance. In a typical enterprise data center, power managment is a major challenge impacting server density and the total cost of ownership (COO). Storage uses a significant fraction of the power budget and there are no widely deployed power-saving solutions for enterprise storage systems. This work presents Data Center Networks (DCNs) for efficient power management in the context of SMART Grids. A SMART DCN is modelled with OPNET 14.5 for Network, Process and Node models. Also, an Extended SMART Integration Module in the context of SMART DCN is shown to be more cost effective than the traditional distribution grid in DCNs. The implementation challenges are discussed also. This paper suggests that smartening the grid for DCN will guarantee a sustainable energy future for the enterprise segments.
\end{abstract}

Keywords- energy; density; enterprise; power budget; framework smart grids.

\section{INTRODUCTION}

Contemporarily, most discussions and professional conferences now focus on the power industry and its resurgent energy challenges. It is a worldwide goal to optimize energy demand, consumption and $\mathrm{CO} 2$ emissions [1] in all critical sectors of any economy. Energy reduction in enterprise setups is a major concern to operators and regulators all over the world. A part of this energy reduction scheme concerns the telecommunication industry and ICT that participates in a direct, indirect and systematic ways [2]. Characteristic examples are green networks, smart buildings, smart grids, Intelligent Transportation Systems (ITS), energy efficient electronics (OLEDS, photonics, nanotechnology) and the application of embedded systems towards low carbon and energy efficient technologies [2],[3].

The IT/Telecommunication industry suffers from myriads of persistent power challenges in the electricity power supply as well as optimizing the available energy reserves. This research suggests that a power solution that will help this critical sector to manage demand growth, conserve energy, maximize asset utilization, cost and improve grid security reliability as well as reduce carbon foot print will go a long way in solving the persistent power issues faced by the enterprise market segments. In our context, a new proposal for power management in an energy efficient data centers (fixed broadband networks) and its challenges form the basis for this paper.

According to [4], a Data Centre is the consolidation point for provisioning multiple services that drive an enterprise business processes. It is also known as the server farm or the computer room. The data center is where the majority of enterprise servers and storage systems are located, operated and managed like the Enterprise resource planning solutions (ERPs), application servers, security systems (IDS). It generally includes redundant or backup power supplies, redundant data communications connections, environmental controls (e.g. Air conditioning, fire suppression) and security devices. DCNs have attracted a lot of interest in today's enterprise network segments [5]. The work in [6] proposed a technology to fix the energy demands for reliable delivery in DCNs. However, the requirement to serve the Data Center environment mandates that a new grid framework with enhanced performance characteristics, hence, this paper proposes SMART GRID model for efficient power management in DCNs.

The paper is organised as follows. Related work is summarised in Section 2. Data center networks and energy requirements are discussed in this section. We presented Smart Grids Proposal for DCNs in Section 3. Smart Grids Reference Model is discussed in Section 4. Section 5 presents a cost effective distribution model for DCNs. Section 6 discussed the challenges of SMART GRID Implementation and finally conclusions and future work are presented.

\section{RELATED WORKS}

Following the definition in [4], various proposals have been presented on how to optimize performance in DCNs. The work done in [1] gave a review of energy efficiency in telecommunication networks while concentrating on data center, fixed line and cellular networks. Representative sample of literatures on DCN traffic, design methodology and management were studied in [6], [7], [8], [9]. According to the EPA report in [7], the two largest consumers of electricity in the data center are: 
- Support infrastructure - $50 \%$ of total.

- General servers - $34 \%$ of total.

Since then, significant strides have been made to improve the efficiency of servers in DCNs. High density blade servers and storage are now offering much more compute capacity per Watt of energy. Server virtualization is allowing organizations to reduce the total number of servers they support, and the introduction of Energy Star servers have all combined to provide many options for both the public and private sectors to reduce that $34 \%$ of electricity being spent on the general servers.

Essentially, the outline in [9], presents a holistic power design considerations for DCNs. According to the EPA, most data centers consume $100 \%$ to $300 \%$ of additional power for the support systems than are being used for their core IT operations. However, through a combination of best practices and migration to fast-payback facility improvements, this overhead can be reduced to about $30 \%$ of the IT load. Within corporations, the focus on power management in data centers is driven primarily by a desire to reduce the tremendous electricity costs associated with operating a data center. Hence, going green is recognized as a way to reduce operating expense significantly for the IT infrastructure.

In general, the telecommunication sector accounts for approximately $4 \%$ of the global electricity consumption [10]. Figure 1 shows a typical DCN.

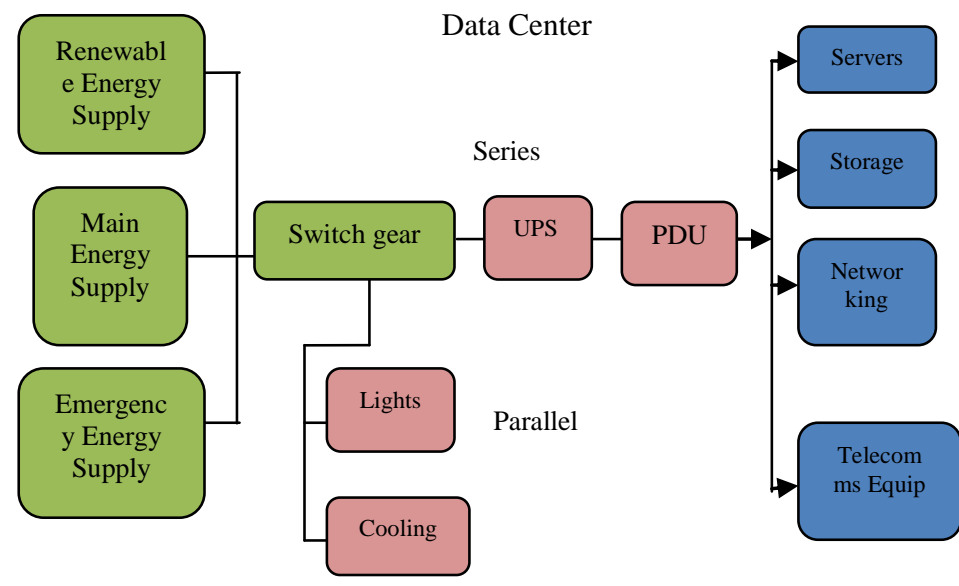

Fig. 1: A Typical Data Center Network

From fig. 1 The power consumption of a data center is related to the associated power consumed by each module in the DCN. Efficiency at individual parts is an important step for 'smartening' the data center but optimization is achieved when the efficiency is aimed at the overall data center design.

The power distribution in a typical data center is divided into an in-series path and an in-parallel path to feed the switchgear and the cooling systems, respectively [1]. Thermal heat at the switchgear, UPS and PDUs, creates losses owing to AC/DC/AC conversions. As shown in fig.1, the parallel path feeds the cooling system for heat protection in the data center. The power consumption at different layers of the data center is presented in Table 2. it is observed that the power consumption pattern in a DCN is dependent on the input workload to the data center and the surrounding environmental characteristics. The authors in [11] presented a power saving estimation scheme; correlation aware power optimization (CARPO) which achieves energy savings mainly by turning off unused switches after conducting correlation-aware consolidation. The work explains that the dynamic power in a DCN is relatively much smaller compared with the static power consumption of the network devices. As such, for a given DCN topology and workloads, it is possible to approximately estimate the energy savings of a DCN using a correlation aware power optimization scheme that dynamically consolidates traffic flows onto a small set of links and switches in a DCN and then shuts down unused network devices for energy savings. By their empirical measurements, the power consumption model of the entire network in their testbed is

given by:

$$
\mathrm{P}=\text { 6.67.Ns }+\sum_{\mathrm{i}=1}^{3}(\mathrm{Pi} . \mathrm{Ji})
$$

where $N s$ is the number of active virtual switches, $P i$ is the active power of a single port at the data rate level $i$ and $J i$ is the corresponding number of active ports at that data rate level.

This work argues that energy redundant devices in a DCN can not be adequately taken care of by CARPO owing various sources of estimation errors in the conventional power management approach. Fig. 2a and Fig. 2b presents DCN network and nodel models for different functionalities of the network .The DCN model was splitted into core, aggregation and access layers. The energy consumption is higher at the access part of the network. Also, at the core layer which provides for data centers computations, storage, applications and data transfer, the drain is considerable. We observed that the backbone (aggregation) networks present lower energy demands.On these basis, this work argues that an energy efficient architecture should focus on intelligent and efficient access techniques that is efficient in its operation. Hence, we propose a SMART grid model with optimized power management. Basically, the main functionalities of the SMART DCN includes regeneration, transportation, storage, routing, switching and processing of data. As ealier stated, the largest part of energy is consumed for routing/switching, regeneration and processing of data. Both communication protocols and electronic devices are responsible for this consumption and this imposes challenges for more sophisticated transport techniques, thermal removal from switches or the servers and less redundant data transfers. A characteristic example of energy efficiency in DCN equipments is shown in Table 1.

The proposed model in this work is self configurable and stable under all load conditions. Since the power consumption of a DCN is largely dependent of its workload, mainly because the network equipment, it now becomes very imperative to adapt SMART grid based DCN so as to put network devices, such as switches and routers, into a sleep state during periods of very inactive traffic activities, and buffer the packets during the inactive period to transmit in future time. This will gurantee return on investment in today's typical multi-tiered DCNs. In this case, high degrees of oversubscription on the network devices will result to good energy savings for the 
DCN. As a result, frequently putting network devices into cold state under low traffic intermittently in a DCN will cause packets to be buffered around the deactivated DCN devices and their paths.
The framework in fig.1 serves as a good energy svaing model but lacks the capacity to handle energy drain in redundant components.

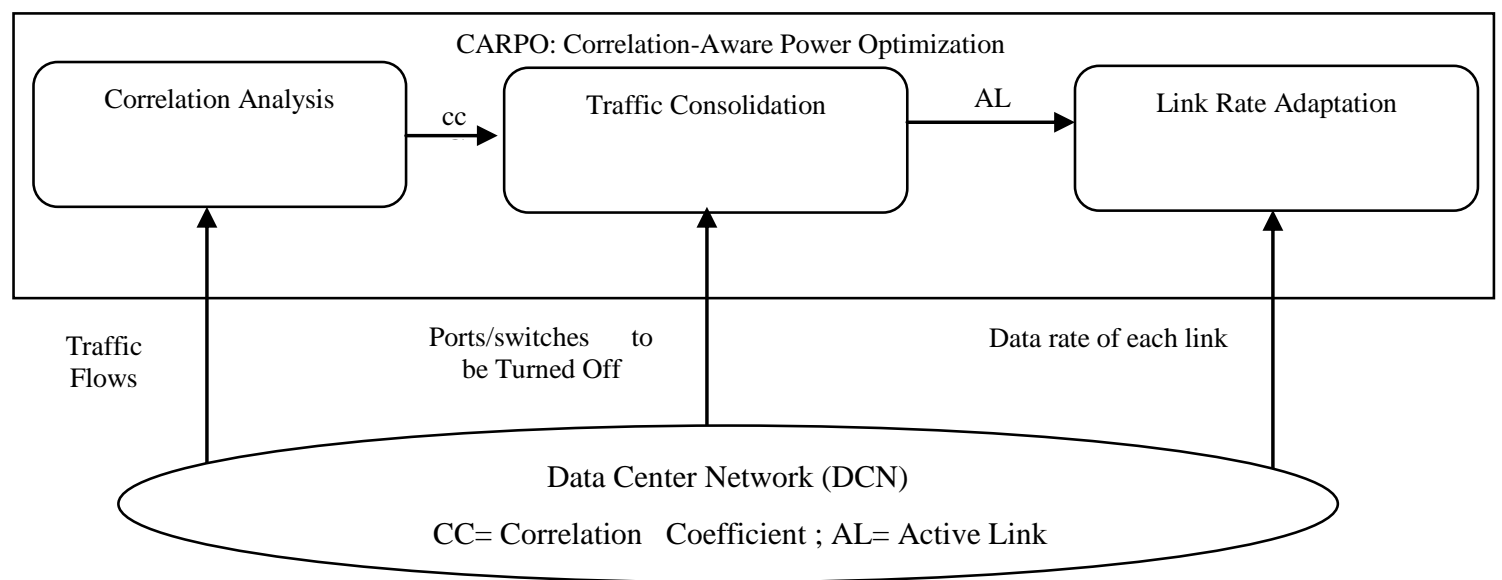

Fig. 1b. The CARPO framework [11].

TABLE 1: POWER EFFICIENCY OF DCN EQUIPMENTS.

\begin{tabular}{|l|l|}
\hline Equipments & Power Efficiency (W/Gbps) \\
\hline Router & 40 \\
\hline IP Switch & 25 \\
\hline Transport TDM & 80 \\
\hline ATM Switch & 80 \\
\hline
\end{tabular}

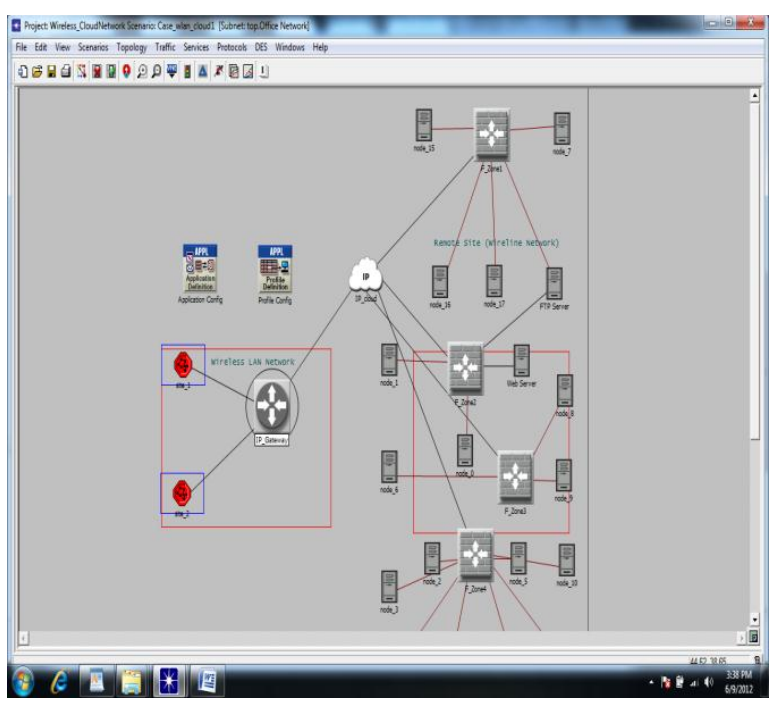

Figure 2a: DCN Model for SMART Grid Integration

The power consumption at different layers of the data center is presented in Table 2 .

It can be observed that the useful work of data center is associated to a percentage of power, smaller than the $30 \%$ delivered to the IT equipments.
In Fig 2a. We modelled a SMART DCN with OPNET modeller 14.5 as well as generating various node and process models for the setup.

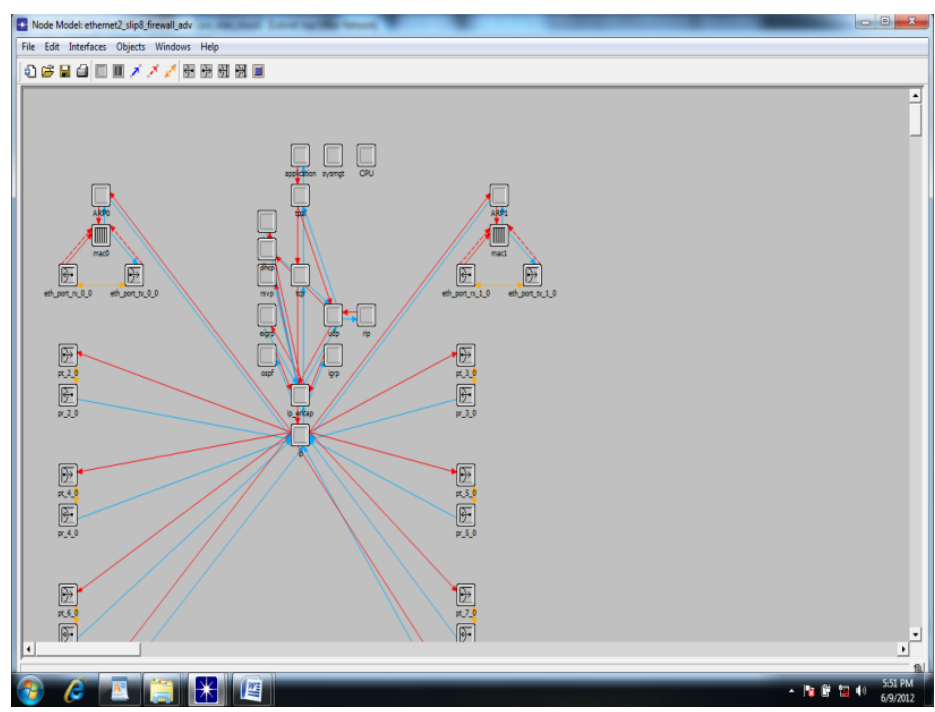

Figure 2b: DCN Process Model for SMART Grid Integration

Since, this work is not centered on performance analysis, we rather observed the system response after configuring the power parameters as well as the traffic parameters.

The proposed model in Fig. 8b, is believed to address energy demands of figure $2 \mathrm{a}$. 
TABLE 2: POWER WASTE DISTRIBUTION IN TYPICAL DATA CENTERS.

\begin{tabular}{|l|l|}
\hline NCPI Equipments & $\begin{array}{l}\text { Percentage of power consumption } \\
\text { [Total 70\%] }\end{array}$ \\
CRAC & 33 \\
UPS & 9 \\
PDU & 19 \\
Switchgear, Light & 5 \\
\hline IT Equipments & 4 \\
System & relative to [Total 30 \%] \\
Disks & 25 \\
Power Supply & 5 \\
Networking & 13 \\
CPU & 9 \\
\hline Memory & 40 \\
\hline
\end{tabular}

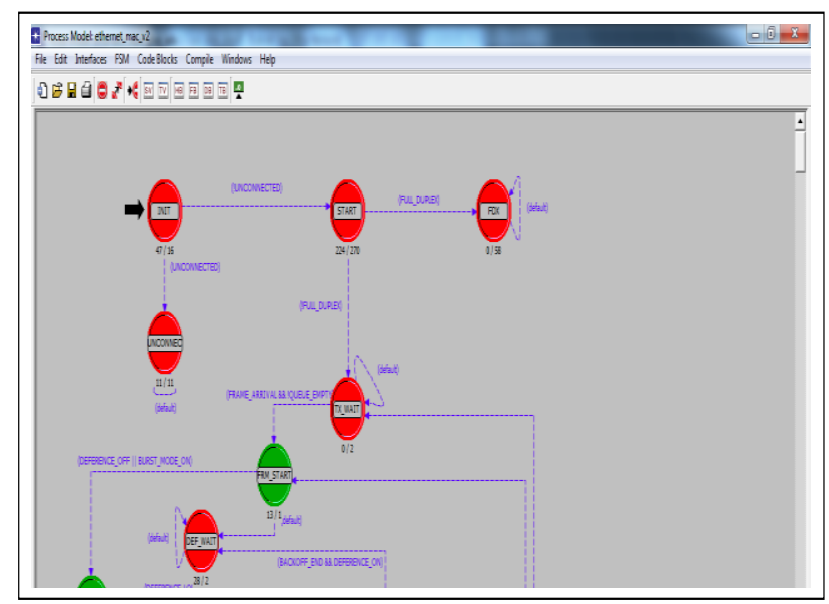

Figure 2c: A Node Model For SMART DCN

\section{ENERGY EFFICIENCY IN DCN}

In a SMART DCN model, configuring a network to operate in an efficient manner is a complex task. However, optimizing energy consumption and total network optimization is considered very critical for power management in DCN. For a network to work in an energy efficient way enhancing environmental sustainability, it must create flexibility for the deployment of future networks to off grid areas that rely on Renewable Energy Sources (RES) and sensor networks that rely on battery power supply. Minimizing power consumption on the DCN will have a great effect on the cost of operation of a network and this makes it more affordable in general.

This paper opions that network optimization in terms of energy efficiency can be achieved by providing the following key metrics viz: efficiency to network dimensioning, efficiency in network processes, efficiency at the access network,etc for better power management of the equipments as shown in Fig.3. Optimization of DCN equipments is the first step for an energy efficient network. This will be taken care of by the SMART DCN architecture which has provision for low power IT devices and equipments, and efficient battery technology. In addition, recycling of equipments is considered as a valuable solution for energy efficiency as well.

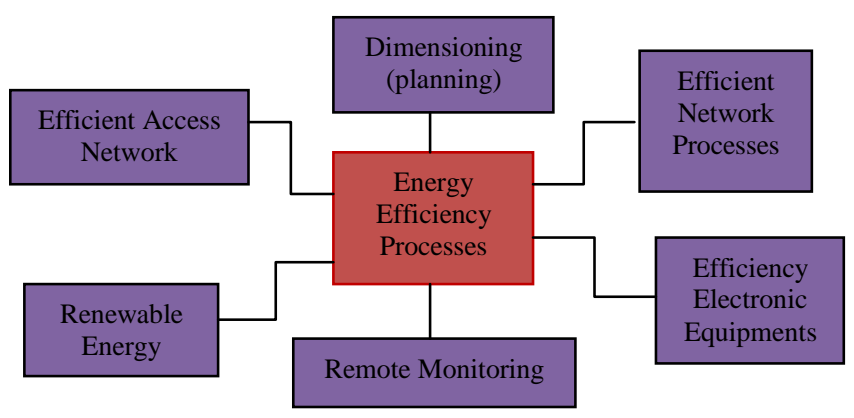

Fig. 3: Main factors of energy efficient networks.

DCNs infrastructure (Data centers and servers) constitute critical components of networks providing data processing, storage, regeneration, etc. A metric for energy efficiency of data center is the Data Center Infrastructure Efficiency (DCIE) and the Data Center energy Productivity (DCeP) [15]. According to [15], DCIE expresses the fraction of the total power supplied to the data center and is delivered to the IT load.

A SMART energy efficient data center requires operational and planning actions that will correspond to the IT equipment needs and incorporate the use of energy proportional servers, such as blade servers with good cooling units. Also, the planning actions again will consider the exploitation of virtualization, remote monitoring and management of the data center, while using vertical rightsizing procedures and actions to reduce cooling needs through optimal design of the DCN domain. Finally, by using SMARD grid architure in DCNs, power management will grantee zero down time.

\section{DETERMINING DATA CENTER CAPACITIES}

In designing the SMART data center capacity, it will involve many different variables that includes: the housing infrastructure, network feeds necessary to keep the DCN functional, the storage and hardware processing units. Balancing all of these variables to design a data center that meets the project scope and keeps the DCN in constant operation is very essential for power management of a planned DCN. According to [9], the design of data centers is dependent on the balance of two critical sets of capacities: 
1. Data center capacities: Power, cooling, physical space, weight load, bandwidth (or connectivity), and functional capacities.

2. Equipment capacities: The various devices (typically equipment in racks) that could populate the data center in various numbers

The knowledge of the equipment requirements was used to determine the size of the center, the amount of power and cooling needed, the weight load rating of the raised floor, and the cabling needed for connectivity to the network. Hence, data center size as well as in-feeds determines how much equipment to be deployed in Fig. 2a. The work in [9] proposes a new method for designing a data center based on the critical capacities called rack location units (RLUs). The actual process of defining RLUs to determine the capacities of a data center boils down to careful planning which enhances flexibility. The Rack Location Unit (RLU) system is a completely flexible and scalable system that can be used to determine the equipment needs for a data center of any size, whether 100 or $100,000,000$ square feet. RLUs are defined based on specific DCN device requirements. These requirements are the specifications that come from the equipment manufacturers. These requirements are:

1. Power (how many outlets/circuits it requires, how many watts it draws)

2. Cooling (BTUs per hour that must be cooled)

3. Physical space (how much floor space each rack needs, including the cooling dimensions)

4. Weight (how much a rack weighs)

5. Bandwidth (how it connects to the network)

Functional capacity (how much computational power, physical memory, disk space, as well as how many spindles, MFLOPS, database transactions, and any other measures of rack functions).

However, this work only seeks to adapt smart grid functionality into DCN for efficient power management (reliability, improvement, loss control, and cost optimization) while presenting its economic implications.

\section{SMART GRIDS PROPOSAL FOR DCNS}

As shown in figure $2 \mathrm{a}$, we seek to adapt smart grid to monitor and manage the transport of electricity from all generation sources to meet the varying electricity demands of the SMART DCN. The Smart grid framework co-ordinates the needs and capabilities of the SMART DCN, minimizing costs and environmental impacts while maximizing system reliability, resilience and stability. In context of this paper, smart grids electricity system (transmission and distribution) powers the storage (SAN) module of the DCN. Fig. 4 and Fig. 5 show our proposed DCN Smart Grid Model and Smart Grid components.

By leveraging on Smart Grids that improves the efficiency, reliability, economics, and environmental sustainability, an efficient Power management in DCN can thus be achieved. The DCNs, with the integration of SMART grids will now :

1. Increase reliability, efficiency and safety of the power grid.
2. Enable decentralized power generation thereby aloowing DCNs effectively manage energy usage.

3. Allow flexibility in power consumption at the DCN's side to allow supplier selection (distributed generation, solar, wind, and biomass).

4. Increase GDP by creating more new, green-collar energy jobs related to renewable energy industry.
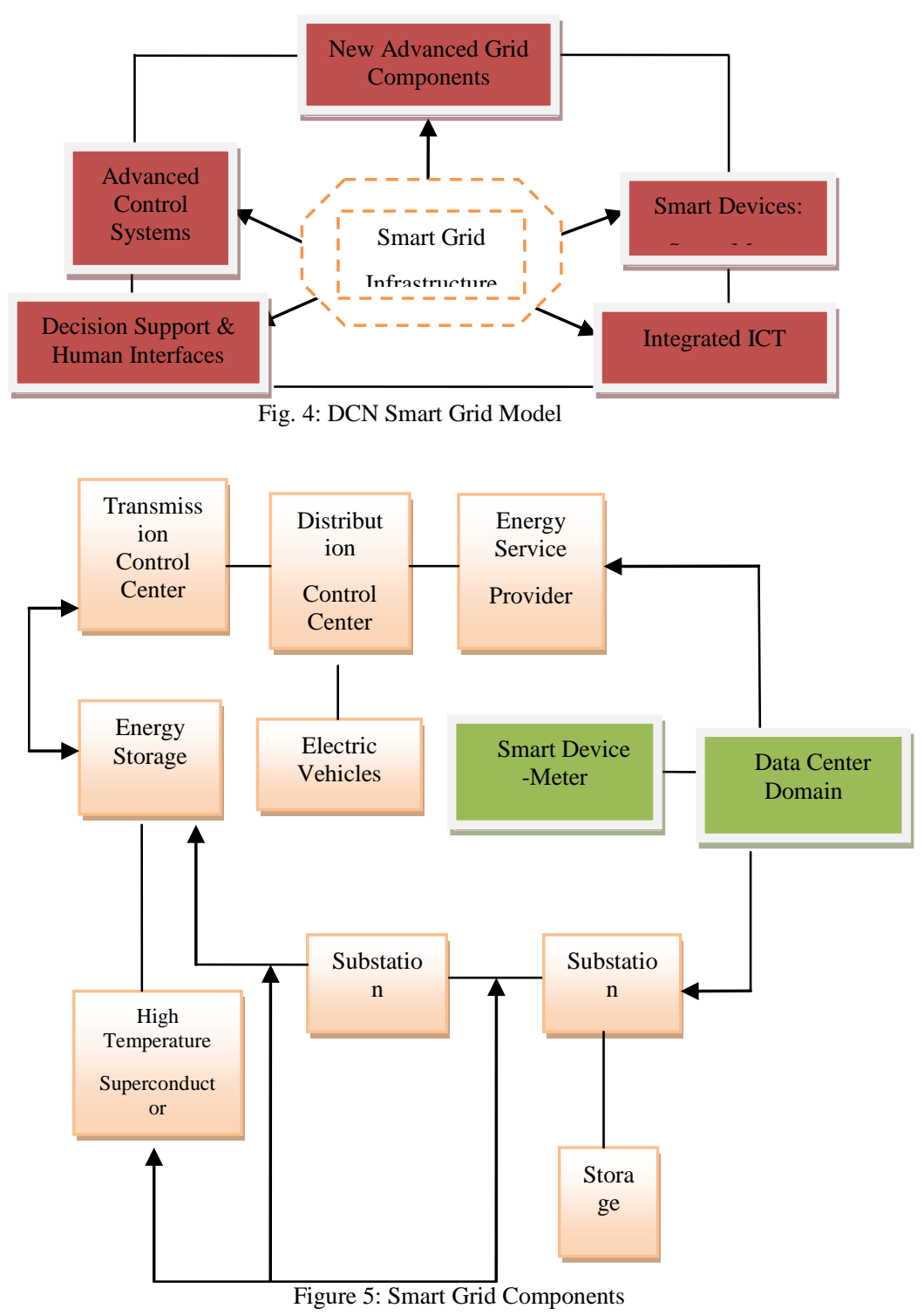

From Fig. 4, this paper recommends that regulators and DCN operators streamlines the technical, financial and regulatory details that will enable the maximization of the potential of smart grids. From Fig. 5, smart grid is a highly complex combination and integration of multiple digital and non-digital technologies and systems which outlines the main component of a smart grids viz : i) new and advanced grid components, ii) smart devices and smart metering, iii) integrated communication technologies, iv) decision support and human interfaces, v) advanced control systems. 
New and advanced grid components allow for efficient energy supply, better reliability and availability of power. The components includes: advanced conductors and superconductors, improved electric storage components, new materials, advanced power electronics as well as distributed energy generation. Superconductors are used in multiple devices along the grid such as cables, storage devices, motors and transformers. The rise of new high-temperature superconductors allows transmission of large amounts of power over long distances at a lower power loss rate to DCNs. Distributed energy can be channelled to the DCN to be served, hence this will improve reliability, and can reduce greenhouse gas emissions while wideing an efficient SMART energy delivery to the DCN.

Smart devices and smart metering include sensors and sensor networks. Sensors are used at multiple places along the grid, e.g. at transformers, substations and at customers/DCNs. They play an outstanding role in the area of remote monitoring and they enable demand-side management and thus new business processes such as real-time pricing. The design technique for digital meters is influenced by three major factors namely; desired device cost, efficiency and overall size [21]. While the cost is influenced by users' general affordability, the efficiency and size must strictly comply with standard. This work proposes the adaptation of Fig. 6 in our DCN Smart grid model.

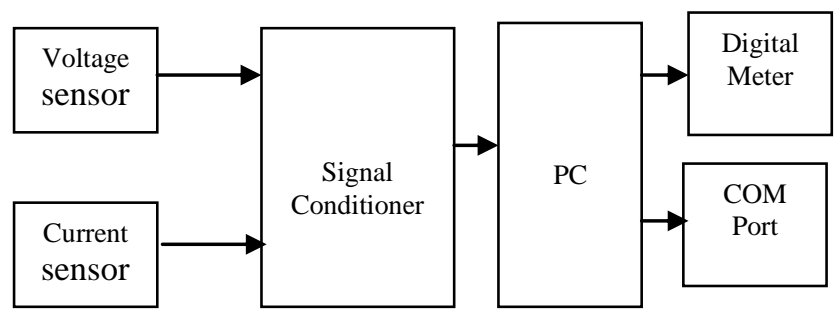

Fig. 6: Block Diagram of Digital Energy (Smart) Meter [21]

\section{SMART GRIDS REFERENCE MODEL}

There are four major layers identified in SG integrations as shown in Fig. 7. The SG decision intelligence (layer-4) comprises of application programmes that run in relays, intelligent electronics, substation automation systems (SAS), control centers (CC) and enterprise zones. These programs process the information collected from the sensors or dispatched from layer-3 (ICTs) and then provide control directives and business process decisions via layer 1 (Power C-T-S-C). The advanced metering infrastructure (AMI) creates a 2-way handshake platform for demand response and other grid distribution roles. Layer-1 is primarily for energy conversion, transmission, storage and consumption. This gives the platform for DCN power interfacing. The traditional grid energy distribution lacks storage, fast reactive and real power regulation and distributed distribution. This accounts for numerous power management challenges in DCN.

As depicted in Fig. 8b, the Extended SMART Integration Module (ExSim) for DCN is presented to address the limitations of Fig. 8a. In addition, it supports grid-feeder connection, AC and DC bus systems, coordination and optimization of energy supply. The Energy storage block houses all available energy options (wind, hydro, and solar PV) which provides for a short to medium-term power supply buffer for uninterrupted DCN operation hence improving reliability and reduction in energy losses.

\section{The CHALLENGeS OF SMART GRID IMPLEMENTAIONS}

A smart grid strategy requires information integration across autonomous systems. From IT perspective, some of it challenges includes: Economic, Political and regulatory policies: The regulators will need to understudy and take into account the load demands in DCNs and come up with environmental friendly green policies. In this context, existing regulatory framework for DCNs needs to take care of future scalability and match consumers intrests at large. Dynamics in Technology in accordance with Moore's Law: Unlike other low carbon energy technologies, smart grids must be deployed in both existing systems (which in some cases are over 40 years old) as well as within totally new systems and it must be deployed with minimum disruption to the daily operation of the electricity system [22]. Also, Despite Moore's laws, most countries still have epilleptic technologies that is still at a very initial stage of development and are yet to be developed to a significant level. As the technologies advances, it will reduce the delivery and full deployment by vendors.

i. With legacy operational systems and business processes, the challenge by stakeholders to endorsement of and upgrade to SMART Grids platforms will have to be addressed conclusively, else this will constitute a major barrier.There is need for DCN operators, and other consumer's orientation to understand power delivery intricacies before venturing into implementation. Smart grid low content carbon economy should be analyzed in the context of environmental sustainability for all stake holders in DCN as well as other users.

ii. The high cost of implementation of smart grid will call for power deregulation to attract investors that will generate funds for its rollout.

$\mathrm{v}$ Technical expertise (Need for extensive training and orientation).

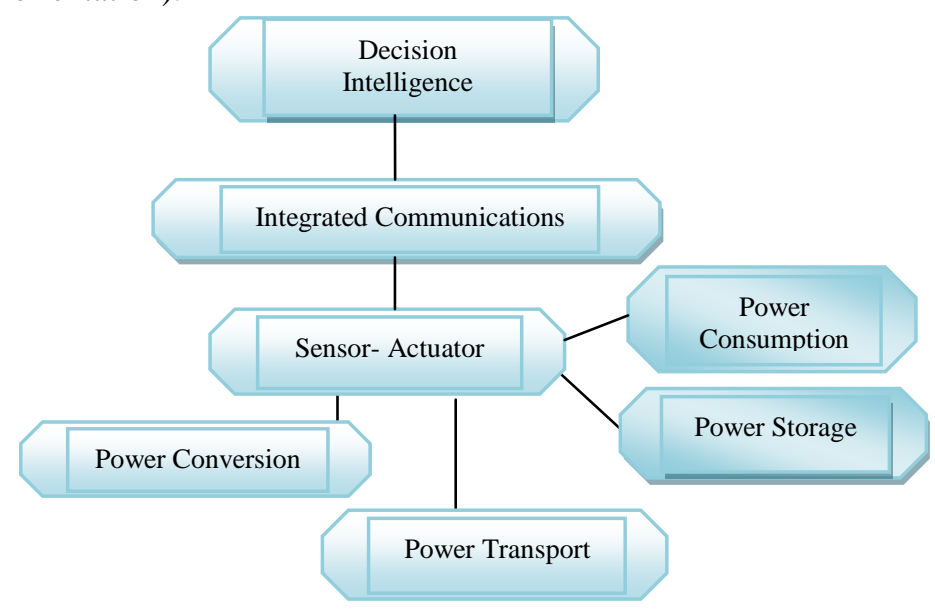

Fig. 7: layers in SG integrations 


\section{Cost EfFective DCN Distribution Model}

Following the limitations of the tradition energy supply for DCNs in Fig. 8a, this work proposes an optimized Smart Grid model for DCNs as shown Fig. 8b.

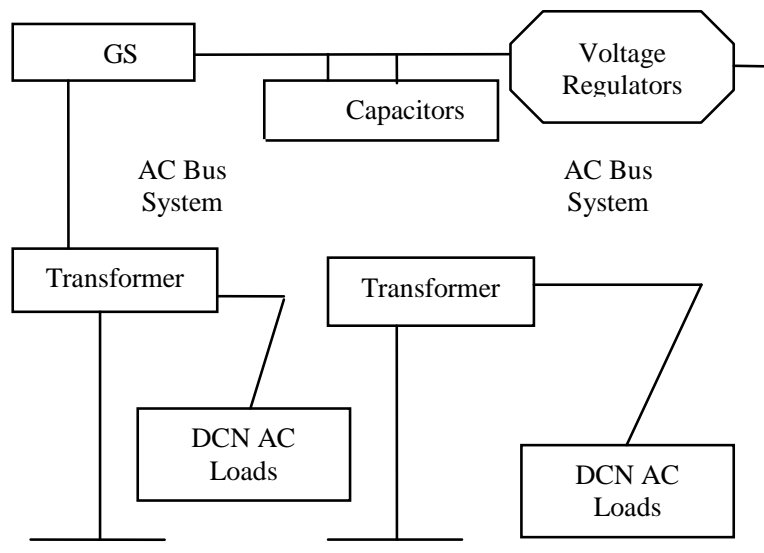

Fig. 8a: Traditional Distribution System in DCN

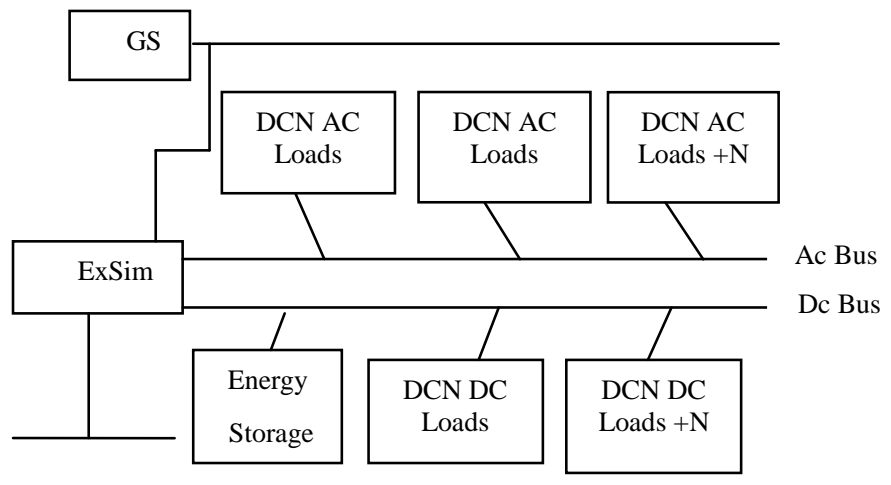

Fig. 8b: Optimized SG model for DCNs (ExSim)

\section{CONCLUSIONS}

This paper proposes SMART Grids as a better alternative to DCN power management. The world's electricity systems face a number of challenges, including ageing infrastructure, continued growth in demand, the integration of increasing numbers of variable renewable energy sources, etc. There is an urgent need to improve the availability, reliability, security of supply as well as lowering carbon emissions for environmental sustainability. Smart grid technologies offer better ways to meet these challenges and also develop a cleaner energy supply that is more energy efficient, more affordable and more sustainable. Also, Extended SMART Integration Module for DCN is proposed as a cost effective SMART GRIDS model. Since the contemporary digital society depends on and demands a power supply of high quality and high availability, implementing SMART Grids for the enterprise market segments yeilds a colossal gain.

\section{FUTURE WORK}

Smart grid is a new technlogy that seeks to revolutionize the current electricity grids into a flexible decentraized service network for operators and clients, future work will ascertain the correlation between SMART grid acceptance index and demand response coefficient. Also, drivers for SMART grids like increase in demand and peak load increase will be investigated. Various model validation experiments will be analysed with MATLAB software in the future work.

\section{REFERENCES}

[1] G. Koutitas, and P. Demestichas, " A Review of Energy Efficiency in Telecommunication Networks”, Telfor Journal, Vol. 2, No. 1, 2010.

[2] Report on Climate Change, International Telecommunication Union (ITU), Oct. 2008.

[3] H. Scheck, "Power consumption and energy efficiency of fixed and mobile telecom networks," ITU-T, Kyoto, 2008.

[4] http://ieee802.org/802_tutorials/Data-Center-Bridging-Tutorial-Nov2007-v2.pdfGigabitEthernet-Wikipedia.

[5] Okafor kennedy, Udeze Chidiebele. C,. C, Prof. H. C. Inyiama, Dr. C.C okezie, "Cloud Computing: A Cost Effective Approach to Enterprise Web Application Implementation (a Case for Cloud ERP Web Model),(Unpublished)

[6] Okafor K.C., "SVLAN Approach to Congestion Management in Data Center Networks", M.Eng Thesis , University of Nigeria, Nsukka, 2010.

[7] A. Greenberg, et al, "Towards a Next Generation Data Center Architecture: Scalability and Commoditization",(Unpublished).

[8] White Paper: Data Center Definition and Solutions.[Online]Available: http://www.cio.com/article/print/499671, August 14, 2009.

[9] R. Snevely, "Enterprise Data Center Design and Methodology", Copyright 2002 Sun Microsystems.

[10] A. Gladisch, C. Lange, R. Leppla, "Power efficiency of optical versus electronic access networks," Proc. European Conference and Exhibition on optical communications, Brussels, 2008.

[11] Xiaodong Wangy, Yanjun Yao, Xiaorui Wangy, Kefa Lu, Qing Cao, "CARPO: Correlation-Aware Power Optimization in Data Center Networks", (Unpublished)

[12] L. A. Barroso, U. Holzle, The data center as a computer: An introduction to the design of warehouse-scale machines, Morgan and Claypool, ISBN:9781599295573, 2009.

[13] N. Rasmussen, "Allocating data center energy costs and carbon to IT users," APC White Paper, 2009.

[14] A.Vukovic, "All-optical Networks - Impact of Power Density", Panel on "Challenges of Electronic and Optical Packaging in Telecom and Datacom Equipment", Maui, Hawaii, USA, July 2003

[15] U.S Environmental Protection Agency ENERGY STAR Program, Report to Congress on Server and Data Center Energy Efficiency, Public Law 109-431, page 94, August 2007.

[16] Smartgrids Advisory Council. "Driving Factors in the Move Towards Smartgrids" (PDF). European Smart grids Technology Platform: Vision and Strategy. European Commission. p. 9.ISBN 92-79-01414-5. http://www.smartgrids.eu/documents/vision.pdf

[17] Smart Sensor Networks: Technologies and Applications for Green Growth, December 2009

[18] Smartgrids Advisory Council. "Driving Factors in the Move Towards Smartgrids" (PDF). European Smartgrids Technology Platform: Vision and Strategy. European Commission. p. 9. ISBN 92-79-01414-5. http://www.smartgrids.eu/documents/vision.pdf.

[19] National Energy Technology Laboratory (2007-07-27) (PDF). A Vision for the Modern Grid. United States Department of Energy. p. 5. Retrieved, 2008-11-27.

[20] DOE (U.S. Department of Energy) (2009), Smart Grid System Report

[21] M.C. Ndinechi, O.A. Ogungbenro, K.C. Okafor, "Digital Metering System: A Better Alternative For Electromechanical Energy Meter In Nigeria" International journal of academic research vol. 3. No.5. September, 2011.

[22] White paper: Technlogy Roadmap. www.iea.org, 2011

\section{AUTHORS PROFILE}

Okafor Kennedy. C. is a Senior R \& D Engineer. He has B.Eng \& M.Engr in Digital Electronics \& Computer Engineering from the University of Nigeria Nsukka, (UNN). He a Cisco expert and currently works with the ICT department of Electronics Development Institute, ELDI. He is a member of IEEE, IAENG and NCS. Email: arissyncline@yahoo.comDr. ECN Okafor is 
a senior lecturer in the electrical electronics department of Federal University of Technology Owerri, Nigeria. He is an expert consultant in power systems engineering. His current research interest is on power systems engineering and smart grid. E-mail: encokafor2000@yahoo.com

Udeze Chidiebele C. received his B.Eng and M.Sc in Electrical Electronics Engineering with Nnamdi Azikwe University, Awka, Nigeria .He is a Senior R \& D Engineer with Electronics development Institute Awka, Nigeria and also a member of NSE. He is currently running his Ph. D program in computer and control systems engineering. His current research interest is on data center networks, wireless sensor networks and control systems engineering. Email: udezechidi@yahoo.com

C.C Okezie is a senior lecturer in the electronic and computer department of Nnamdi Azikiwe University Awka. She received her B. Eng, M. Eng, and Ph.D from Enugu State University of Science and Technology, Enugu, Nigeria. She majors in digital systems and control engineering and has many publications to her credit. Email- christianaobioma@yahoo.com. 